\title{
TI.95.1
}

\section{Baseline Expectations for Trust in Federation: Increasing Trust and Interoperability in InCommon}

- PDF: TI.95.1-Baseline_Strategy.pdf

- Text:TI.95.1-Baseline_Strategy.txt

\section{More Information}

\begin{tabular}{|l|l|}
\hline Repository ID & TI.95.1 \\
\hline Persistent URL & http://doi.org/10.26869/TI.95.1 \\
\hline Title & Baseline Expectations for Trust in Federation: Increasing Trust and Interoperability in InCommon \\
\hline Authors & Tom Barton, Brett Bieber, Ann West, Dean Woodbeck \\
\hline Sponsor & Ann West, Associate Vice President, Trust and Identity, Internet2 \\
\hline Review & \\
\hline Status & Preserve \\
\hline Publish Date & January 10,2018 \\
\hline DOI & $10.26869 /$ TI.95.1 \\
\hline Signature & \\
\hline Deprecated & no \\
\hline Future Review & December 2019 \\
\hline Supersedes & none \\
\hline Format & \\
\hline Related Docs & \\
\hline Development Location & \\
\hline IP Framework & \\
\hline Subject Tags & InCommon Federation, Assurance, CTAB, Baseline Expectations, Trust \\
\hline Notes & \\
\hline
\end{tabular}

